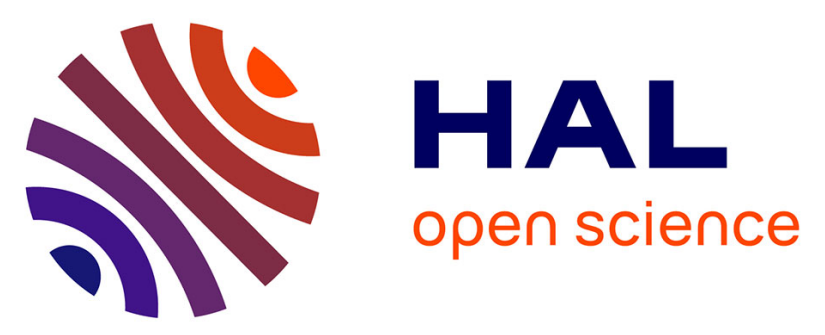

\title{
High-Precision FLIM-FRET in fixed and living cells reveals heterogeneity in a simple CFP-YFP fusion protein
}

Michael Millington, G. Joan Grindlay, Kirsten Altenbach, Robert K. Neely, Walter Kolch, Mojca Benèina, Nick D. Read, Anita C. Jones, David T.F. Dryden, Steven W. Magennis

\section{To cite this version:}

Michael Millington, G. Joan Grindlay, Kirsten Altenbach, Robert K. Neely, Walter Kolch, et al.. High-Precision FLIM-FRET in fixed and living cells reveals heterogeneity in a simple CFP-YFP fusion protein. Biophysical Chemistry, 2007, 127 (3), pp.155. 10.1016/j.bpc.2007.01.008 . hal-00501645

\section{HAL Id: hal-00501645 \\ https://hal.science/hal-00501645}

Submitted on 12 Jul 2010

HAL is a multi-disciplinary open access archive for the deposit and dissemination of scientific research documents, whether they are published or not. The documents may come from teaching and research institutions in France or abroad, or from public or private research centers.
L'archive ouverte pluridisciplinaire HAL, est destinée au dépôt et à la diffusion de documents scientifiques de niveau recherche, publiés ou non, émanant des établissements d'enseignement et de recherche français ou étrangers, des laboratoires publics ou privés. 


\section{Accepted Manuscript}

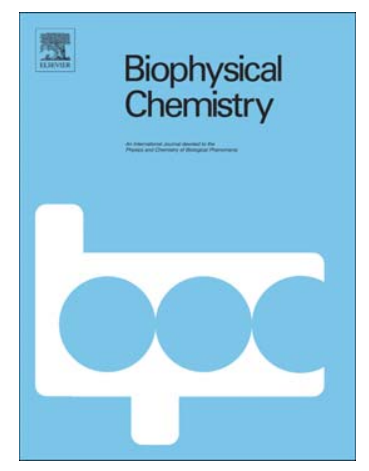

High-Precision FLIM-FRET in fixed and living cells reveals heterogeneity in a simple CFP-YFP fusion protein

Michael Millington, G. Joan Grindlay, Kirsten Altenbach, Robert K. Neely, Walter Kolch, Mojca Benèina, Nick D. Read, Anita C. Jones, David T.F. Dryden, Steven W. Magennis

PII: $\quad$ S0301-4622(07)00018-X

DOI: doi: $10.1016 /$ j.bpc.2007.01.008

Reference: $\quad$ BIOCHE 4926

To appear in: Biophysical Chemistry

Received date: $\quad 5$ January 2007

Revised date: 23 January 2007

Accepted date: $\quad 24$ January 2007

Please cite this article as: Michael Millington, G. Joan Grindlay, Kirsten Altenbach, Robert K. Neely, Walter Kolch, Mojca Benèina, Nick D. Read, Anita C. Jones, David T.F. Dryden, Steven W. Magennis, High-Precision FLIM-FRET in fixed and living cells reveals heterogeneity in a simple CFP-YFP fusion protein, Biophysical Chemistry (2007), doi: $10.1016 /$ j.bpc.2007.01.008

This is a PDF file of an unedited manuscript that has been accepted for publication. As a service to our customers we are providing this early version of the manuscript. The manuscript will undergo copyediting, typesetting, and review of the resulting proof before it is published in its final form. Please note that during the production process errors may be discovered which could affect the content, and all legal disclaimers that apply to the journal pertain. 


\title{
High-Precision FLIM-FRET in fixed and living cells reveals heterogeneity in a simple CFP-YFP fusion protein
}

\author{
Michael Millington ${ }^{[a, b]}$ G. Joan Grindlay, ${ }^{[\mathrm{d}}$ Kirsten Altenbach, ${ }^{[\mathrm{a}, \mathrm{d}]}$ Robert K. Neely, ${ }^{[\mathrm{a}, \mathrm{b}}$ \\ Walter Kolch, ${ }^{[\mathrm{e}, e]}$ Mojca Bencina,${ }^{[]]}$Nick D. Read,${ }^{[\mathrm{a}, \mathrm{d}]}$ Anita C. Jones, ${ }^{[\mathrm{a}, \mathrm{b}]}$ David T. F. \\ Dryden, ${ }^{[a, b]}$ and Steven W. Magennis* ${ }^{[a, b]}$
}

[a] Collaborative Optical Spectroscopy, Micromanipulation and Imaging Centre (COSMIC), The University of Edinburgh

King's Buildings, Edinburgh EH9 3JZ (UK)

[b] School of Chemistry, The University of Edinburgh

King's Buildings, Edinburgh EH9 3JJ (UK)

[c] The Beatson Institute for Cancer Research, Garscube Estate, Switchback Road, Bearsden, Glasgow, G61 1BD (UK)

[d] Fungal Cell Biology Group, Institute of Molecular Plant Sciences, The University of Edinburgh, King's Buildings, Edinburgh EH9 3JH (UK)

[e] Sir Henry Wellcome Functional Genomics Facility

University of Glasgow, Glasgow G12 8QQ (UK)

[f] Laboratory of Biotechnology, National Institute of Chemistry Hajdrihova 19, SI-1000 Ljubljana (Slovenia)

*Corresponding author:

Dr. Steven W. Magennis

Fax: +44 (0) 131650 5902; Tel: +44 (0) 131650 5229; Email: s.magennis@ed.ac.uk 


\begin{abstract}
We have used widefield photon-counting FLIM to study FRET in fixed and living cells using control FRET pairs. We have studied fixed mammalian cells expressing either cyan fluorescent protein (CFP) or a fusion of CFP and yellow fluorescent protein (YFP), and living fungal cells expressing either Cerulean or a Cerulean-Venus fusion protein. We have found the fluorescence behaviour to be essentially identical in the mammalian and fungal cells. Importantly, the highprecision FLIM data is able to reproducibly resolve multiple fluorescence decays, thereby revealing new information about the fraction of the protein population that undergoes FRET and reducing error in the measurement of donor-acceptor distances. Our results for this simple control system indicate that the in vivo FLIM-FRET studies of more complex protein-protein interactions would benefit greatly from such quantitative measurements.
\end{abstract}

Keywords: CFP, YFP, fluorescence, lifetime, proteins 


\section{Introduction}

The goal of proteomics is a full molecular-level understanding of the cellular functioning of protein molecules [1]. This requires the study of the spatio-temporal distribution and behaviour of proteins in vivo. In particular, knowledge of the nanoscale interaction of proteins with other cellular components (including other proteins) is essential. Fluorescence microscopy has proven to be an invaluable method for imaging in cell biology, primarily due to its high sensitivity and the wide range of probes available for the selective labelling of cellular structures [2]. While conventional fluorescence microscopy is ultimately limited in the spatial resolution achievable (diffraction limited to $\sim 200 \mathrm{~nm}$ ), spatial resolution can be improved by using point scanning methods such as confocal microscopy [3], and other sophisticated microscopy developments such as multiphoton, stimulated emission depletion, 4-Pi, image interference and near-field scanning microscopy [4].

An alternative approach is to take advantage of the phenomenon of Förster resonance energy transfer (FRET) [5]. This involves monitoring the transfer of energy from an excited fluorescent molecule (donor) to another molecule (acceptor). The efficiency of energy transfer is proportional to the inverse sixth power of the distance between donor and acceptor, making it extremely sensitive to interactions in the $1-10 \mathrm{~nm}$ range [5].

Whilst the choice of potential fluorophores is considerable, the most important for in vivo fluorescence studies in recent years has been the green fluorescent protein (GFP), which is an inherently fluorescent recombinant probe [6]. A wide range of GFP mutants have been engineered to extend and optimize their physicochemical properties. In particular, a number of 
colour variants have been constructed for use as complementary donor-acceptor pairs for FRET studies [7]. Although these probes have made an enormous impact on cell biology, extracting quantitative information is non-trivial. Quantification is particularly difficult with steady-state fluorescence imaging because variations in intensity can be due to local environmental effects (such as energy transfer), changes in the probe concentration or a range of optical artefacts.

Fluorescence lifetime imaging (FLIM), which spatially maps the donor lifetime [8], can overcome the problems of intensity-based methods because the lifetime is independent of the number of fluorescing molecules, so changes in lifetime reflect changes in the environment of the probe (e.g. proximity of an acceptor chromophore, ion concentration or temperature). FLIM has been successfully used to probe energy transfer in cells [9-11]. However, the multiple decay pathways available to an excited molecule due to variations in local environment or chemical structure (e.g. due to tautomerisation, protonation or ion binding) mean that the fluorescence decay is often complex. Such dynamic complexity must be taken into consideration when extracting quantitative information from FRET measurements, and this is an emerging area of research [12-16].

In this paper, we report the use of the wide-field time-domain FLIM technique of time- and space-correlated single photon counting (TSCSPC) [17] to investigate the effect of the photophysical complexity of popular GFP-derived FRET probes, expressed in fixed mammalian and living fungal cells, on the amount of information that may be extracted. The cells express either a cyan variant of GFP or a fusion protein of the cyan variant with a short amino acid linker to a yellow variant of GFP. This constrains the two fluorophores to be in close proximity and allows FRET to occur. For the mammalian cells, the cyan variant is ECFP (called CFP henceforth) and the yellow variant is EYFP (called YFP henceforth). YFP is attached to CFP by 
a two-amino-acid linker. The fungal cells express either mCerulean (called Cerulean henceforth), which is a relatively new cyan variant [18] or Cerulean linked by an eight-amino-acid linker to Venus, an improved variant of YFP [19].

We show that the high-precision photon-counting data reveal complex fluorescence decays in fixed and living cells. We show that an accurate measurement of FRET in cells requires the quantitative analysis of complex decays in terms of multiple fluorescence lifetimes. The use of average lifetimes can result in a significant underestimation of FRET efficiency and donoracceptor separation. In this work, we determine for the first time the donor and acceptor ratio of FRET interactions in cells using FLIM.

\section{Experimental Section}

Plasmids used for mammalian expression: eCFP-C1 was from BD-Clontech. pcDNA3-CFP-YFP was a gift from G. Milligan (IBLS, Glasgow University). YFP was inserted as a Kpn1/Not1 fragment in pcDNA3 (Invitrogen). CFP was added between HindIII and Kpn1. The expressed protein has a two amino acid linker (Gly-Thr) between the last amino acid of CFP and the first amino acid of YFP. All plasmid constructs were verified by DNA sequencing (AGOWA,GmBH).

Mammalian cell culture and transfection: Cos-1 cells (ATCC CRL-1650) derived from African green monkey kidney fibroblasts were maintained in DMEM/10\% fetal calf serum (Invitrogen). $10^{5}$ cells were seeded in 6-well dishes and transfected with $0.5 \mu \mathrm{g}$ plasmid DNA using Effectene (Invitrogen). After 18 hours, cells were washed in PBS, trypsinised and replated on sterile glass 
coverslips, then protein expression was allowed for a further 20-24 hours in fresh medium before fixation in $3.7 \%$ paraformaldehyde and cold methanol [20]. Coverslips were mounted on glass slides using Vectashield (VectorLabs H-1000) and stored in the dark. Data were recorded within 2-3 days.

Construction of plasmids $\mathrm{p} 9 \mathrm{mCer}$ and $\mathrm{p} 9 \mathrm{CeVe}-\mathrm{N}$ used for fungal expression: DNA containing the gene for the mCerulean protein was kindly provided by D. W. Piston (Vanderbilt University, U.S.A.) and for the Venus protein by A Miyawaki (RIKEN Brain Science Institute, Japan). To construct p9mCer, mCerulean was amplified by PCR using the following oligonucleotides: mCerulean 213 5'- ccatggtgagcaagggcga-3' (NcoI) and 183 5'-gaattcttacttgtacagctcgtccatgcc-3' (EcoRI). The resulting PCR fragment, digested with NcoI and EcoRI, was introduced into the fungal expression vector pMOJ009 under control of the A. nidulans gpdA promoter and trpC terminator over the NcoI/EcoRI sites.

To construct p9CeVe-N, mCerulean and Venus were amplified by PCR using the following oligonucleotides: mCerulean 213 5'- $\quad$ ccatggtgagcaagggcga-3' $\quad$ (NcoI) and $185 \quad 5^{\prime}$ gaattcgcatgcgggcggcggtcacgaactcc-3' $\quad$ (EcoRI, $\quad$ SphI); $\quad$ Venus $\quad 184 \quad$ 5'ggatcccgcatgcatgggggcaccggtgggtccgagctcatggtgagcaagggcgagg-3' (BamHI, SphI, SstI) and 183 5'-gaattcttacttgtacagctcgtccatgcc-3' (EcoRI), leading to the introduction of the stated oligonucleotides at the 5' ends. The resulting PCR fragments, digested with NcoI and SphI (mCerulean fragment) or SphI and EcoRI (Venus fragment) were introduced into pMOJ009 over the $\mathrm{NcoI} / \mathrm{SpHI} / \mathrm{EcoRI}$ sites. In the expressed $\mathrm{CeVe}-\mathrm{N}$ protein, mCerulean and Venus are separated by an eight amino acid linker with sequence MHGGSGGTE. 
Fungal cell culture and transformation: $100 \mathrm{ml}$ of liquid $\mathrm{CM}$ medium, supplemented with final concentrations of leucin $(0.2 \mathrm{~g} / \mathrm{l})$, nicotinamide $(1 \mathrm{mg} / \mathrm{l})$ and uridine $(5 \mathrm{mM})$ were inoculated with $10^{6}$ spores of the Aspergillus niger strain 455 and incubated for 16 hours at $30{ }^{\circ} \mathrm{C}$ in a shaking incubator. Protoplasts were prepared after harvesting the mycelium by treatment with 10 $\mu \mathrm{g} / \mathrm{ml}$ Caylase C4 (Cayla, Toulouse, France) and transformed as described by Kusters-van Someren et al. [21]. Prior to imaging, coverslips were inoculated with $100 \mu$ l of the A. niger spore suspension and incubated for 16 hours at $30^{\circ} \mathrm{C}$.

Fluorescence imaging: The excitation source was a Ti-Sapphire femtosecond laser system from Coherent (10W Verdi and Mira Ti-Sapphire laser) producing pulses of $200 \mathrm{fs}$ at $76 \mathrm{MHz}$. The output of the Mira was passed through a pulse picker (reducing the repetition rate to $4.75 \mathrm{MHz}$ ) and then frequency doubled to give an output at $420 \mathrm{~nm}$ (live cells) or $430 \mathrm{~nm}$ (fixed cells). The excitation beam was split, and one portion was used to trigger a fast photodiode. The photodiode output was passed through a constant fraction discriminator $(\mathrm{CF} 4000$, Ortec), and used as the trigger signal.

The Ti-Sapphire laser beam was expanded, collimated and directed into a Nikon TE300 inverted microscope operating in an epifluorescence configuration. This excitation light was reflected from a dichroic mirror (DM455, Nikon) mounted in a standard filter cube and focussed onto the sample using either 100× (PF, NA=1.3, oil immersion, Nikon), 60× (PA, NA=1.2, water immersion, Nikon) or $40 \times(\mathrm{PF}, \mathrm{NA}=1.3$, oil immersion, Nikon) objectives. The laser power incident on the entry port of the microscope was typically around $2 \mu \mathrm{W}$. The resultant fluorescence was collected through the same objective, passed through a barrier filter (465-495 $\mathrm{nm}$ for cyan donor; 510-560 nm for yellow acceptor) and imaged onto the FLIM detector (see below). For initial location of the cells, the laser was replaced with a $\mathrm{Hg}$ lamp (Nikon, $\mathrm{LH}$ - 
M100CB-1) using a 410-420nm excitation filter and DM455 dichroic (Nikon) for cyan fluorescence and a 460-500 nm excitation filter, DM505 dichroic (Nikon) and 505-560 nm emission filter for yellow fluorescence. Transmission images were recorded using the TE300 condenser and a Nikon camera (DXM1200 using ACT-1 software control).

Fluorescence emission from the sample was imaged using the technique of time and space correlated single photon counting (TSCSPC) using a quadrant anode detector from Europhoton $\mathrm{GmbH}$ (Berlin). The data acquisition was in reverse mode, with each detected photon assigned to one of 4096 channels, each of width 27 ps. Typically the count rate over the entire detector was

$30 \mathrm{kHz}$, giving an acquisition time of $\sim 15$ minutes. Areas of the image were selected and the lifetime decay data extracted and analyzed. Data analysis was performed with F900 software (Edinburgh Instruments, Livingston, UK), via a tail-fitting procedure from the peak of the decay. The instrument response of the system, measured using a mirror, was 400 ps FWHM, and no improvement in fits was found by reconvoluting with the instrument response. Families of decays (e.g. from different regions of interest of cells of the same type) were analysed globally using Alango's FAST software (Edinburgh Instruments), i.e. they were fitted simultaneously, with lifetimes, $\tau_{\mathrm{i}}$, as common parameters. The counts in the peak channel were typically around $10^{4}$. The quality of fit was judged on the basis of the reduced chi-square statistic, $\chi^{2}$, and the randomness of residuals. The lifetime maps were generated using QA Analysis software (Europhoton $\mathrm{GmbH}$ ), fitting from the peak of the fluorescence decay. The background was estimated by taking the mean counts per pixel (cpp) of areas of background and subtracting this level from the entire image. A lifetime map was then produced by assigning a colour on a 16-bit pseudocolour scale to a fitted single exponential decay time, and these were displayed over a range of 0-3.2 ns. 
Distance estimation from crystal structures: The donor-acceptor distances in fusions of cyan and yellow proteins were estimated using structures from the RCSB protein data bank (www.rcsb.org/pdb). The cyan protein was ECFP (code 1CV7.pdb) and the yellow protein was EYFP (1YFP.pdb). The structures were studied using DeepView/Swiss-Pdb Viewer v 3.7 (www.expasy.org/spdbv).

\section{Results}

Cos-1 mammalian cells expressing CFP alone or a CFP-YFP fusion protein, and living fungal cells (germinated spores) of Aspergillus niger expressing Cerulean or a Cerulean-Venus fusion were studied using TSCSPC. The TSCSPC data was used to generate a lifetime map, by fitting data from each point in the field of view to a single exponential decay; typical lifetime maps are shown in Fig. 1. Cells that express the same fluorescent protein have similar lifetimes with little intracellular variation. Uniform lifetime distribution is expected since the CFP fluorophore is not fused to any mammalian protein or directed to any specific cellular location. There is a consistent drop in the average lifetime of CFP between the CFP cells (Fig. 1a) and the CFP-YFP cells (Fig. 1b); there is a similar drop in the average lifetime of Cerulean between the Cerulean (Fig. 1c) and the Cerulean-Venus cells (Fig. 1d). This decrease in lifetime is attributed to FRET between the two fluorescent proteins that are closely tethered by a short amino acid linker in the donoracceptor constructs. 
The lifetime map represents an approximate lifetime and only displays the true fluorescence decay when it is monoexponential. However, TSCSPC can also be used to detect and quantify complex decay behaviour in cells, which can potentially provide much more information about the FRET process. Fig. 2 shows typical TSCSPC data for mammalian cells expressing CFP (Fig. 2a) or CFP-YFP (Fig. 2b), and fungal cells expressing either Cerulean (Fig. 2c) or CeruleanVenus (Fig. 2d), together with decays from various regions of interest (ROIs), as indicated on the fluorescence intensity image. The decays, which have been normalised to allow easy comparison, clearly show that there is little variation between different parts of the same mammalian cell and that Cerulean or Cerulean-Venus expressed in living fungal cells display qualitatively similar dynamics to the CFP and CFP-YFP in fixed mammalian cells.

To quantify the decay processes in these cells, the data were fitted to a multiexponential decay function (Eq. 1):

$$
I(t)=\sum_{i=1}^{n} A_{i} \exp \frac{-t}{\tau_{i}}+B
$$

where $\tau_{\mathrm{i}}$ is the fluorescence lifetime, $A_{i}$, the "A-factor", is the fractional amplitude of the $i^{\text {th }}$ decay component, and $B$ is the background. The A-factor indicates the fraction of the emitting molecules that has a particular lifetime, $\tau_{\mathrm{i}}$. As will be demonstrated later, the A factors of the donor decay enable the fraction of donors undergoing FRET to be determined. The resolution and accuracy of the analysis is improved by simultaneously fitting all of the ROIs of a particular cell type [22]. The results of this global analysis for the mammalian and fungal cells are shown in Table 1, giving the globally fitted lifetimes, mean A-factors, and the global $\chi^{2}$ values. Details 
of the individual A-factors and local $\chi^{2}$ values from global analysis are shown in Supplementary Table 1.

The global analysis of CFP shows that the decay is well fitted by a biexponential function with lifetimes of $1.36 \mathrm{~ns}$ and $3.04 \mathrm{~ns}$, and A-factors of $39 \pm 8 \%$ and $62 \pm 6 \%$, respectively. For cells expressing CFP-YFP, three lifetimes are required to give excellent fits with lifetimes of $0.65 \mathrm{~ns}, 1.65 \mathrm{~ns}$ and $3.11 \mathrm{~ns}$, and mean A-factors of $20 \pm 9 \%, 50 \pm 7 \%, 30 \pm 10 \%$, respectively. The lifetimes of the two long components in CFP-YFP are essentially the same as those seen in the CFP cells; the fractional contribution of one remains essentially unchanged, while the contribution of the longer one ( $\sim 3 \mathrm{~ns})$ is halved. The additional lifetime component is the shortest of the three. A comparison between the 2- and 3-exponential fits from a typical CFP-YFP cell is shown in Fig. 3. The residuals for the 2-exponential fit have an obvious systematic deviation, which disappears in the 3-exponential fit, and there is a concomitant decrease in the $\chi^{2}$ value.

Previous time-resolved studies have also found that the fluorescence lifetime of CFP in mammalian cells is biexponential. Tramier et al. [23] found that the lifetime of CFP in live cells was $1.30 \mathrm{~ns}$ and $3.84 \mathrm{~ns}$, with A-factors of $53 \%$ and 47\%, Duncan et al. [24] measured biexponential decays of $0.42 \mathrm{~ns}(38 \%)$ and $2.19 \mathrm{~ns}(62 \%)$, while Becker et al. [25] measured the two components of CFP in cells to be 1.2-1.3 ns ( 40\%) and 2.8-2.9 ns ( 60\%). For CFP in solution, Rizzo et al. measured the decay to be biexponential with $1.2 \mathrm{~ns}$ and $3.6 \mathrm{~ns}$ lifetimes and A-factors of $14 \%$ and $86 \%$ [18], while Borst et al. [26] fitted two lifetimes of 1.14 ns (33.5\%) and 3.72 ns (66.5\%). Habuchi et al. [27] found evidence for a third short lifetime component (0.24 ns) for CFP in sodium phosphate-buffered saline solutions, though this made a small contribution to the overall decay (1\%), with the biggest contribution for lifetimes of $1.1 \mathrm{~ns}$ 
$(\sim 10 \%)$ and $3.3 \mathrm{~ns}(89 \%)$. The origin of these two lifetime components has been attributed to the presence of two different conformations of the chromophore in CFP due to interactions with nearby amino acids [28]. There is evidence from NMR spectroscopy that these two conformations interconvert on a millisecond timescale [29], which is a much slower timescale than the fluorescence decay. Each conformer will, therefore, exist as a distinct emitting species.

In other photon-counting FLIM studies of FRET between CFP and YFP in cells, the extent of FRET has been described by biexponential kinetics, with two lifetimes that are different to the donor-only decays [23-25]. More complex kinetics were not observed. Biexponential decays were also observed for fusions of Cerulean with the yellow acceptor mCit [18]. TSCSPC allows much higher photon counts to be acquired than conventional photon-counting FLIM (confocal or multiphoton), which we believe has allowed us to discern the greater complexity of the dynamics. Becker et al. suggested the possibility of a third lifetime when CFP and YFP take part in FRET, though they were unable to discriminate between the two and three-component fits in the cells they studied [25].

The results of global analysis for the fungal cells are remarkably similar to those found for the mammalian cells expressing CFP and CFP-YFP, despite the fact that Cerulean and Venus are different fluorescent protein variants and are expressed in completely different cells. The global analysis of Cerulean returns a biexponential decay with lifetimes of 1.97 ns and 3.65 ns, with Afactors of $51 \pm 3 \%$ and $49 \pm 3 \%$, respectively, while the data for the Cerulean-Venus fusion protein fits three lifetimes of $0.72 \mathrm{~ns}, 1.99 \mathrm{~ns}$ and $4.26 \mathrm{~ns}$, with A-factors of $40 \pm 6 \%, 51 \pm 3 \%, 9$ $\pm 3 \%$, respectively. Not only do the absolute lifetimes match those of the mammalian cells, but the populations of each species are of similar magnitudes. There is a twofold larger population 
with a subnanosecond lifetime in Cerulean-Venus than in the CFP-YFP fusion protein, and a concomitantly smaller population with a $\sim 4$ ns lifetime. Notably, the population of the $<2$ ns component is exactly the same (51\%) in cells expressing the Cerulean alone or the fusion, mirroring the trend observed for the mammalian cells.

Whilst the biexponential decay of CFP is now established, Rizzo et al. reported that Cerulean displayed simpler fluorescence decays that were best described by monoexponential kinetics [18]. However, these authors reported that fitting the Cerulean decays to a two-exponential decay model with lifetimes of $2.3 \mathrm{~ns}(38 \%)$ and $4.5 \mathrm{~ns}(62 \%)$ improved the $\chi^{2}$ from 2.3 to 1.4. Our data show definitively that Cerulean, like CFP, shows a biexponential decay. An independent check was made by diluting fungal cell extracts in buffer and measuring the fluorescence lifetimes in a fluorescence spectrometer, which had high temporal resolution (5-10 ps). Extracts of cells that expressed Cerulean displayed biexponential kinetics with lifetimes of $1.41 \mathrm{~ns}$ and $3.33 \mathrm{~ns}$, each with populations of $55 \%$ and $45 \%$ respectively at an emission wavelength of $470 \mathrm{~nm}$. Fluorescence decays of cell extracts of the fusion protein required 3 lifetime components, with lifetimes of $0.35 \mathrm{~ns}(51 \%), 1.50 \mathrm{~ns}(43 \%)$ and $3.42 \mathrm{~ns}(26 \%)$. These are very similar to the results found in intact living cells. The decays are all faster in the cell extract, but this minor difference can be attributed to the effect of the buffer medium. For example, intracellular refractive index variations will alter the lifetime of the fluorescent proteins, [26,30]. A recent report also comments on the necessity to fit 3 decay components to CFP-YFP bulk TCSPC data in cells, although the decay parameters were not reported [12]. In the absence of crystallographic evidence, we presume that the chromophore in Cerulean can adopt two conformations, similar to the CFP. Therefore, in terms of decay complexity, Cerulean is not an improvement over CFP. 
However, the markedly higher quantum yield and extinction coefficient, and increased resistance to photobleaching of Cerulean do make it an improved chromophore for FRET [18].

One of the benefits of using FLIM to probe FRET, is that it is not necessary to measure fluorescence from the acceptor, only from the donor. This is particularly advantageous for fluorescent proteins, where the available FRET pairs have significant spectral overlap. However, if the acceptor emission can be spectrally resolved it should be possible to measure its rise time which will be identical to the lifetime of those donor molecules that transfer energy to the acceptor. We measured the acceptor fluorescence using TSCSPC to verify the occurrence of FRET. YFP fluorescence of mammalian cells expressing CFP-YFP was detected in the 510-560 $\mathrm{nm}$ range, eliminating most of the CFP fluorescence. The images in Fig. 4 show a cell expressing the CFP-YFP fusion through the CFP emission filter set (a) and the YFP emission set (b) together with the lifetime decays from both images. The YFP emission displays a single exponential decay of 2.69 ns with a rise time of 0.53 ns. The YFP emission of the CeruleanVenus fungal cell extracts was also measured and displayed a single exponential decay of $3.06 \mathrm{~ns}$ and a rise of $0.51 \mathrm{~ns}$. The observation of a rise shows that there has been energy transfer from CFP, although the overlap of the YFP fluorescence with residual CFP emission, and the difficulty in accurately measuring rise times, preclude a more detailed analysis.

\section{Discussion}

We now address the key issue as to whether the new information gained by resolving more complex decays using TSCSPC significantly changes the interpretation of the data and the FRET parameters extracted, compared with the typical approach of measuring average lifetimes. To investigate this, we relate the lifetime of the donor in the donor-only construct $\left(\tau_{\mathrm{D}}\right)$ and the donor 
lifetime in the donor-acceptor fusion $\left(\tau_{\mathrm{DA}}\right)$ to the energy transfer efficiency, $\mathrm{E}$, (Eq. 2). The donor-acceptor distance, $r$, can be calculated using the measured efficiency and the Förster distance, $\mathrm{R}_{0}$, at which energy transfer efficiency is $50 \%$ (Eq. 3). Since the donor and acceptor proteins are linked by a flexible polypeptide linker, we have assumed that the orientation factor, $\kappa^{2}$, is $2 / 3$ [6]. The homogeneity of lifetimes within and between cells suggests that the relative orientation of the donor and acceptor is dynamically averaged. Even if the assumption of $\kappa^{2}=$ $2 / 3$ is invalid, the relative values of the distances calculated below should be correct. We are currently adapting our FLIM experiment to simultaneously measure the time-resolved anisotropy, which will address the issue of orientation factor and its effect on absolute distance measurements.

$$
\begin{gathered}
E=1-\frac{\tau_{A A}}{\tau_{D}} \\
r=R_{0} \frac{1}{E}-1^{1 / 6}
\end{gathered}
$$

We have measured three donor decay times for the fusions, two of which $\left(\tau_{1}\right.$ and $\left.\tau_{2}\right)$ are very similar to those of the donor (Table 1). We label the long-lifetime and short-lifetime conformations of the cyan donor protein as $\mathrm{D}_{1}$ and $\mathrm{D}_{2}$, respectively, and assign the lifetimes as $\tau_{\mathrm{D} 1}$ and $\tau_{\mathrm{D} 2}$, respectively. The value of $\mathrm{A}_{2}$ (the contribution of $\mathrm{D}_{2}$ to the emission) is essentially unchanged in the fusion, compared with the free donor, whereas the value of $A_{1}$ is decreased. 
The decrease in $A_{1}$ is exactly balanced by the new contribution $\left(A_{3}\right)$ from the third decay component.

It has been demonstrated that the protonated chromophore of YFP cannot act as a FRET acceptor [31]. Therefore, in constructing a model to interpret our decay data, we assume that the yellow protein in our fusions can exist in two different forms; one can participate in energy transfer by acting as an acceptor $\left(\mathrm{AC}_{1}\right)$, whilst the other cannot $\left(\mathrm{AC}_{2}\right)$. When the cyan donor undergoes energy transfer to the yellow acceptor $\left(\mathrm{AC}_{1}\right)$, the new cyan protein lifetime is labelled $\tau_{\mathrm{DAl}}$ when $\mathrm{D}_{1}$ is the donor and $\tau_{\mathrm{DA} 2}$ when $\mathrm{D}_{2}$ is the donor. When the cyan donors are fused to the protonated form of the yellow protein, $\mathrm{AC}_{2}$, the donor lifetimes are unchanged.

We believe that there are two ways to interpret our data for FRET analysis, as illustrated by the models in Fig. 5 and the decay times in Table 2. The first model is labelled " $3 \tau$ ". This is the simplest interpretation of the data and assumes that only one of the two donor conformations $\left(D_{1}\right)$ participates in energy transfer. Thus, both the lifetime and A-factor of $\mathrm{D}_{2}$ are unchanged in the fusion because none of the $\mathrm{D}_{2}$ population undergoes FRET. The persistence of $\tau_{\mathrm{Dl}}$ in the fusion, with a reduced A-factor, shows that only a fraction of the population of $\mathrm{D}_{1}$ undergoes FRET to give the new short decay time $\tau_{\mathrm{DAl}}$. The $\mathrm{A}$ factor of $\tau_{\mathrm{DAl}}\left(\mathrm{A}_{3}\right.$ in Table 1 ) thus corresponds to the decrease in the $\mathrm{A}$ factor of $\tau_{\mathrm{DI}}$. This model requires an absence of FRET for one conformation. Whilst this is possible, the flexibility of the polypeptide linking the donor and acceptor proteins and the apparent similarity in position of the two conformations within the protein barrel [28] lead us to propose a second interpretation of the data. 
The second model is labelled " $4 \tau$ " in Table 2. Borst et al. briefly discussed that each of the two CFP decay components might have an associated FRET decay component [26]. In this case, we assume that both conformations participate in FRET, but that one of the unquenched donor lifetimes, $\tau_{\mathrm{D} 2}$, is so similar to one of the donor-acceptor lifetimes, $\tau_{\mathrm{DAl}}$, that they cannot be resolved and appear as a single lifetime, $\tau_{2}$, in the fitted decay function. In other words, there are actually four decay components, but only three can be resolved. Thus, the A-factor $\left(\mathrm{A}_{2}\right)$ of $\tau_{2}$ (Table 1) remains unchanged in the fusion because the increase in A-factor of $\tau_{\mathrm{DAl}}$, as result of FRET from $\mathrm{D}_{1}$ to $\mathrm{AC}_{1}$, is exactly balanced by the decrease in A-factor of $\tau_{\mathrm{D} 2}$, as a result of FRET from $\mathrm{D}_{2}$ to $\mathrm{AC}_{1}$.

In both models, the fraction of unquenched donor fluorescence in the fusion, due to donor bound to $\mathrm{AC}_{2}$, is the same and is given by the value of $\mathrm{A}_{1}$, the $\mathrm{A}$-factor of $\tau_{\mathrm{Dl}}$, in the fusion. In the majority of the cells expressing the CFP-YFP fusion, there is $\sim 30 \%$ of unquenched donor, while cells containing the Cerulean-Venus constructs have $\sim 9 \%$ unquenched donor. The mammalian cells were maintained and fixed in solutions buffered to $\mathrm{pH}$ 7.4. From the reported pKa of EYFP of around 6.9 [19], there should be a $24 \%$ proportion of protonated at $\mathrm{pH}=7.4$, which is in good agreement. The pKa of the Venus chromophore is reported to be 6.0[19]. At pH of 7.6, which is a reasonable estimate for the $\mathrm{pH}$ in fungal cells [32], there would be $2.5 \%$ of protonated acceptor.

Taking the uncertainty in the actual intracellular $\mathrm{pH}$ values into account, all of the unquenched donor fluorescence can be explained by an equilibrium between protonated and unprotonated acceptor. We have assumed that all of the fluorescent proteins in the cells are identical. In principle, it is possible that some of the unquenched donor is also due to a small 
fraction of the protein population in which the acceptor is not expressed or is incorrectly folded. However, since we do not see smaller protein products on a Western blot, and since misfolded proteins are normally degraded in the cell, we do not believe that such proteins are likely to be a significant component of our cells.

The energy-transfer parameters for each model are calculated using Eqs. 2 and 3 and are presented in Table 2. For the $4 \tau$ model, we must make separate calculations for each of the two distinct FRET pairs, hence there are two sets of each parameter, which are given the subscript 1 or 2 , according to whether they are associated with donor $\mathrm{D}_{1}$ or $\mathrm{D}_{2}$, respectively. We contrast the results for the two models with that of the conventional approach of using average lifetimes of the donor-only construct and fusion construct for $\tau_{\mathrm{D}}$ and $\tau_{\mathrm{DA}}$ respectively. This is labelled "average" in Table 2. The value of $\mathrm{R}_{0}$ that is conventionally used in Eq. 3 is derived from steadystate spectroscopic measurements and is an average of the $\mathrm{R}_{0}$ value for each distinct donor and acceptor pair that exists in a sample. In our case, the yellow protein exists in two forms, but only one participates in FRET. The single decay time of YFP fluorescence, shown in Fig 4, confirms this. However, the donor exists in two forms, so $\mathrm{R}_{0}$ is an average value for two donor-acceptor pairs ( $\mathrm{D}_{1}-\mathrm{AC}_{1}$ and $\mathrm{D}_{2}-\mathrm{AC}_{1}$ in Fig. 5).

We observed that the relative contributions of the two decay components of the Cerulean protein in solution were independent of emission wavelength, in agreement with experimental studies [27] and recent quantum mechanical calculations of CFP [33]. Therefore, the only unknown in calculating $\mathrm{R}_{0}$ for the two donor-acceptor pairs is the donor quantum yield. It is reasonable to assume that the radiative rates of the donors $\mathrm{D}_{1}$ and $\mathrm{D}_{2}$ will be similar, and that the quantum yield of each donor conformation will be proportional to the measured decay time for 
that conformation. Therefore the $\mathrm{R}_{0}$ for each donor-acceptor pair can be calculated as follows [34]:

$$
R_{0 i}=\overline{R_{0 i}}\left(\tau_{i} / \bar{\tau}\right)^{1 / 6}
$$

where $R_{0 i}$ is the value of $\mathrm{R}_{0}$ for the $i$ th donor decay (i.e. $R_{01}$ is for $\mathrm{D}_{1}-\mathrm{AC}_{1}$ and $R_{02}$ is for $\mathrm{D}_{2}-\mathrm{AC}_{1}$ ) and $\overline{R_{0}}$ is the average measured from steady-state spectroscopy.

The donor-acceptor distances for each FRET pair have been calculated using these individual values of $\mathrm{R}_{0}$, and the results are shown in Table 2. The $\mathrm{R}_{0}$ figure of $50 \AA$ for CFP-YFP [6,15] is used for the calculation of CFP-YFP and the value of $54 \AA$ for Cerulean-Venus [15].

The clearest outcome from Table 2 is that the average method overestimates the distance because it includes fluorescence from unquenched donor proteins. There is a marked increase in the efficiency of energy transfer in going from the average measurement to the 3-component model for both types of cells. The efficiencies for the 4-component model are less than for the 3component model, but they are still considerably smaller than those for the average method. The differences are most marked for the mammalian cells. The differences between the different methods are smaller for the fungal cells because there is less unquenched donor in the CeruleanVenus system.

Estimates of the absolute distance between the chromophores in the CFP-YFP fusion protein have been made by a simple approach using published crystallographic data. Crystal structure data for ECFP and EYFP were used to estimate the distances from the centre of the chromophore 
to each of the termini, giving values of $25.1 \AA$ for CFP and $23.2 \AA$ for YFP. Together with the two amino acid linker, this gives a reasonable estimate of $50 \AA$ for the donor-acceptor distance. In comparison, the average lifetime method calculated the distance to be $62 \AA$, whilst the $3 \tau$ model gave a distance of $42 \AA$. The $4 \tau$ model, which is the most physically plausible, gave the best match, with distances of $53 \AA$ and $44 \AA$. Differences in distances for the two FRET pairs in the $4 \tau$ model are likely due to uncertainties in the lifetimes (particularly the middle lifetime, which is probably the average of two closely-spaced lifetimes).

\section{Conclusion}

FLIM is a powerful qualitative tool for reliably detecting the occurrence of FRET, as has been demonstrated by many research groups worldwide. However, FLIM methods that measure average lifetimes will only be fully quantitative in the absence of heterogeneity in the fluorescence decays. In particular, fluorescence from unquenched donor molecules severely compromises the ability of average lifetime measurements to make quantitative assessment of donor-acceptor distances and binding stoichiometries. In contrast, we have demonstrated that widefield photon-counting FLIM can allow the accurate measurement of fluorescence decays with at least three components. This has allowed us to quantify the fraction of donors undergoing FRET, and make a more accurate measurement of the donor-acceptor distance.

We have made quantitative FRET measurements of control FRET systems using FLIM with very low excitation power, albeit with relatively long acquisition times. Many FRET experiments require much faster imaging. While photon-counting methods are viewed as being comparatively 
slow, the development of new photon-counting detectors, associated electronics and analysis software is underway by a number of research groups, so it is anticipated that this technique will be extended to real-time cellular imaging of molecular interactions that are occurring in vivo.

This study also emphasizes the need for GFP variants with monoexponential decays, and simple absorption behaviour. However, even when donors with simple decay kinetics are used, complexity may arise due to the presence of donors that do not participate in FRET or, indeed, because of a greater range of donor-acceptor distances. In these situations, the ability to accurately resolve and assign the individual decay components will be essential. Therefore, we believe that high-precision FLIM offers new possibilities for the quantitative use of fluorescent proteins in cell biology.

\section{Acknowledgements}

We wish to thank G. Milligan, D. W. Piston and A. Miyawaki for the gifts of pcDNA3-CFPYFP, mCerulean and Venus, respectively, and A. Robnik for assisting with the fungal cell preparation. This work was supported by the Scottish Higher Education Funding Council (SHEFC), the Engineering and Physical Sciences Research Council (EPSRC), the Biotechnology and Biological Sciences Research Council (BBSRC), the Medical Research Council (MRC), Cancer Research UK, a European Union FP6 Interaction Proteome grant, and the jointly-funded RASOR programme (SHEFC, EPSRC, BBSRC and MRC). 


\section{References}

[1] E. Phizicky, P. I. H. Bastiaens, H. Zhu, M. Snyder, S. Fields, Protein analysis on a proteomic scale, Nature 422 (2003) 208-215.

[2] EJ. W. Lichtman, J.-A. Conchello, Fluorescence microscopy, Nat. Methods 2 (2005) 910919.

[3] J. W. Lichtman, J.-A. Conchello, Optical sectioning microscopy, Nat. Methods 2 (2005) 920-931.

[4] Y. Garini, B. J. Vermolen, I. T. Young, From micro to nano: recent advances in highresolution microscopy, Curr. Op. Biotech. 16 (2005) 3-12.

[5] P. R. Selvin, The renaissance of fluorescence resonance energy transfer, Nat. Struct. Biol. 7 (2000) 730-734.

[6] R. Y. Tsien, The Green Fluorescent Protein, Annu. Rev. Biochem. 67 (1998) 509-544.

[7] R. Heim, R. Y. Tsien, Engineering green fluorescent protein for improved brightness, longer wavelengths and fluorescence resonance energy transfer, Curr. Biol. 6 (1996) 178182.

[8] R. M. Clegg, O. Holub, C. Gohlke, Fluorescence lifetime-resolved imaging: measuring lifetimes in an image, Methods Enzymol. 360 (2003) 509-542. 
[9] T. W. J. Gadella Jr., T. M. Jovin, Oligomerization of epidermal growth receptor factors on A431 cells studied by time-resolved fluorescence imaging microscopy-a stereochemical model for tyrosine kinase receptor activation, J. Cell. Biol. 129 (1995) 1543-1558.

[10] T. Ng, A. Squire, G. Hansra, F. Bornancin, C. Prevostel, A. Hanby, W. Harris, D. Barnes, S. Schmidt, H. Mellor, P. I. H. Bastiaens, P. J. Parker, Imaging protein kinase Ca activation in cells, Science 283 (1999) 2085-2089.

[11] F. G. Haj, P. J. Verveer, A. Squire, B. G. Neel, P. I. H. Bastiaens, Imaging sites of receptor dephosphorylation by $\mathrm{PTB} 1 \mathrm{~B}$ on the surface of the endoplasmic reticulum, Science 295 (2002) 1708-1711.

[12] R. Grailhe, F. Merola, J. Ridard, S. Couvignou, C. Le Poupon, J.-P. Changeux, H. Laguitton-Pasquier, Monitoring protein interactions in the living cell through the fluorescence decays of the cyan fluorescent protein, Chem. Phys. Chem. 7 (2006) 14421454.

[13] W. Zeng, H. E. Seward, A. Málnási-Csizmadia, S. Wakelin, R. J. Woolley, G. S. Cheema, J. Basran, T. R. Patel, A. J. Rowe, C. R. Bagshaw, Resonance energy transfer between green fluorescent protein variants: complexities revealed with myosin fusion variants, Biochemistry 45 (2006) 10482-10491.

[14] S. Pelet, M. J. R. Previte, P. T. C. So, Comparing the quantification of Förster resonance energy transfer measurement accuracies based on intensity, spectral, and lifetime imaging, J. Biomed. Optics 11 (2006) 1-11. 
[15] M. A. Rizzo, G. Springer, K. Segawa, W. R. Zipfel, D. W. Piston, Optimization of pairings and detection conditions for measurement of FRET between cyan and yellow fluorescent proteins, Microsc. Microanal. 12 (2006) 238-254.

[16] M. Tramier, M. Zahid, J.-C. Mevel, M.-J. Masse, M. Coppey-Moisan, Sensitivity of $\mathrm{CFP} / \mathrm{YFP}$ and $\mathrm{GFP} / \mathrm{mCherry}$ pairs to donor photobleaching on FRET determination by fluorescence lifetime imaging microscopy in living cells, Microscop. Res. Tech. 63 (2004) 58-66.

[17] V. Emiliani, D. Sanvitto, M. Tramier, T. Piolot, Z. Petrasek, K. Kemnitz, C. Durieux, M. Coppey-Moisan, Low-intensity two-dimensional imaging of fluorescence lifetimes in living cells, Appl. Phys. Lett. 83 (2003) 2471-2473.

[18] M. A. Rizzo, G. H. Springer, B. Granada, D. W. Piston, An improved cyan fluorescent protein variant useful for FRET, Nature Biotechnol. 22 (2004) 445-449.

[19] T. Nagai, K. Ibata, E. S. Park, M. Kubota, K. Mikoshiba, A. Miyawaki, A variant of yellow fluorescent protein with fast and efficient maturation for cell-biological applications, Nature Biotechnol. 20 (2002) 87-90.

[20] R. M. Brock, I. H. L. Hamelers, T. M. Jovin, Comparison of fixation protocols for adherent cultured cells applied to a GFP fusion protein of the epidermal growth factor receptor, Cytometry 35 (1999) 353-362.

[21] M. A. Kusters-van Someren, J. A. M. Harmsen, H. C. M. Kester, J. Visser, Structure of the Aspergillus niger pelA gene and its expression in the Aspergillus niger and Aspergillus nidulans, Curr. Genet. 20 (1991) 293-299.

[22] J. M. Beechem, E. Gratton, M. Ameloot, J. R. Knutson and L. Brand in: Topics in Fluorescence Spectroscopy, ed. J. R. Lakowicz (Plenum Press, 1991) p. 241-305. 
[23] M. Tramier, I. Gautier, T. Piolot, S. Ravalet, K. Kemnitz, J. Coppey, C. Durieux, V. Mignotte, M. Coppey-Moisan, Picosecond-hetero-FRET microscopy to probe proteinprotein interactions in live cells, Biophys. J. 83 (2002) 3570-3577.

[24] R. R. Duncan, A. Bergmann, M. A. Cousin, D. K. Apps, M. J. Shipston, Multidimensional time-correlated single photon counting (TCSPC) fluorescence lifetime imaging microscopy (FLIM) to detect FRET in living cells, J. Microscop. 215 (2003) 1-

12.

[25] W. Becker, A. Bergmann, M. A. Hink, K. König, K. Benndorf, C. Biskup, Fluorescence lifetime imaging by time-correlated single-photon counting, Microscop. Res. Tech. 63 (2004) 58-66.

[26] J. W. Borst, M. A. Hink, A. van Hoek, A. J. W. G. Visser, Effects of refractive index and viscosity on fluorescence and anisotropy decays of enhanced cyan and yellow fluorescent proteins, J. Fluoresc. 15 (2005) 153-160.

[27] S. Habuchi, M. Cotlet, J. Hofkens, G. Dirix, J. Michiels, J. Vanderleyden, V. Subramaniam, C. C. De Schryver, Resonance energy transfer in a calcium concentrationdependent Cameleon protein, Biophys. J. 83 (2002) 3499-3506.

[28] J. H. Bae, M. Rubini, G. Jung, G. Wiegand, M. H. J. Seifert, M. K. Azim, J.-S. Kim, A. Zumbusch, T. A. Holak, L. Moroder, R. Huber, N. Budisa, Expansion of the genetic code enables design of a novel "gold" class of green fluorescent proteins, J. Mol. Biol. 328 (2003) 1071-1081.

[29] M. H. J. Seifert, D. Ksiazek, P. Smialowski, M. K. Azim, N. Budisa, T. A. Holak, Slow exchange in the chromophore of a green fluorescent protein variant, J. Am. Chem. Soc. 124 (2002) 7932-7942. 
[30] K. Suhling, J. Siegel, D. Phillips, P. M. W. French, S. Lévêque-Fort, S. E. D. Webb, D. M. Davis, Imaging the Environment of Green Fluorescent Protein, Biophys. J. 83 (2002) 3589-3595.

[31] M.-A. Elsliger, R. M. Wachter, G. T. Hanson, K. Kallio, S. J. Remington, Structural and spectral response of green fluorescent protein variants to changes in $\mathrm{pH}$, Biochemistry 38 (1999) 5296-5301.

[32] S. J. A. Hesse, G. J. G. Ruitjer, C. Dijkema, J. Visser, Intracellular pH homeostasis in the filamentous fungi Aspergillus niger, Eur. J. Biochem. 269 (2002) 3485-3494.

[33] I. Demachy, J. Ridard, H. Laguitton-Pasquier, E. Durnerin, G. Vallverdu, P. Archirel, B. Lévy, Cyan Fluorescent Protein: Molecular Dynamics, Simulations, and Electronic Absorption Spectrum, J. Phys. Chem. B 109 (2005) 24121 -24133.

[34] S. Albaugh, R. F. Steiner, Determination of distance distribution from time domain fluorometry, J. Phys. Chem. 93 (1989) 8013-8016. 


\section{Tables}

\section{Table 1}

Global analysis of TSCSPC data from mammalian and fungal cells. The individual A-factors and local $\chi^{2}$ values from global analysis are shown in Supplementary Table 1. Errors are standard deviations from global analysis

\begin{tabular}{|c|c|c|c|c|c|c|c|c|}
\hline Cell Type & Construct & $\tau_{1} / \mathrm{ns}$ & $\tau_{2} / \mathrm{ns}$ & $\tau_{3} / \mathrm{ns}$ & $A_{1}$ & $\mathrm{~A}_{2}$ & $\mathrm{~A}_{3}$ & global $\chi^{2}$ \\
\hline \multirow{2}{*}{ Mammalian } & CFP & 3.04 & 136 & $\downarrow$ & $0.62 \pm 0.06$ & $0.38 \pm 0.06$ & $\downarrow$ & 1.10 \\
\hline & CFP-YFP & 3.11 & 1.65 & 0.65 & $0.30 \pm 0.10$ & $0.50 \pm 0.07$ & $0.20 \pm 0.09$ & 1.19 \\
\hline \multirow{2}{*}{ Fungal } & Cerulean & 3.65 & 1.97 & $\downarrow$ & $0.49 \pm 0.03$ & $0.51 \pm 0.03$ & $\downarrow$ & 1.06 \\
\hline & Cerulean-Venus & 4.26 & 1.99 & 0.72 & $0.09 \pm 0.03$ & $0.51 \pm 0.03$ & $0.40 \pm 0.06$ & 1.17 \\
\hline
\end{tabular}




\section{Table 2}

FRET analysis of TSCSPC data from mammalian and fungal cells. The donor lifetimes $\left(\tau_{\mathrm{D}}\right)$, donor-acceptor lifetimes $\left(\tau_{\mathrm{DA}}\right)$, FRET efficiencies $(\mathrm{E})$, Förster distances $\left(\mathrm{R}_{0}\right)$ and donor-acceptor distances (r) are given. See text and Figure 5 for details of the different FRET models. The lifetimes are from global analysis. The errors in the efficiencies and distances were propagated from the standard deviations in lifetimes extracted from single decay analyses of the data (not shown).

\begin{tabular}{|c|c|c|c|c|c|c|c|c|c|c|c|}
\hline Cell Type & $\begin{array}{l}\text { FRET } \\
\text { method }\end{array}$ & $\begin{array}{l}\tau_{\mathrm{D} 1} \\
/ \mathrm{ns}\end{array}$ & $\begin{array}{l}\tau_{\mathrm{DA} 1} \\
/ \mathrm{ns}\end{array}$ & $\begin{array}{l}\tau_{\mathrm{D} 2} \\
\mathrm{lns}\end{array}$ & $\begin{array}{l}\tau_{\mathrm{DA} 2} \\
/ \mathrm{ns}\end{array}$ & $\mathrm{E}_{1} / \%$ & $\mathrm{E}_{2} / \%$ & $\mathrm{R}_{01} / \AA$ & $\mathrm{R}_{02} / \AA$ & $\mathrm{r}_{1} / \AA$ & $\mathrm{r}_{2} / \AA$ \\
\hline \multirow{3}{*}{ Mammalian } & 3- $\tau$ & 3.11 & 0.65 & 1.65 & $\downarrow$ & $79.1 \pm 4.6$ & $\downarrow$ & 52.2 & $\downarrow$ & $41.8 \pm 1.9$ & $\downarrow$ \\
\hline & $4-\tau$ & 3.11 & 1.65 & 1.65 & 0.65 & $47.0 \pm 7.1$ & $60.6 \pm 9.9$ & 52.2 & 47.0 & $53.3 \pm 2.5$ & $43.7 \pm 3.0$ \\
\hline & Av erage & 2.40 & 1.89 & $\downarrow$ & $\downarrow$ & $21.3 \pm 10.2$ & $\downarrow$ & 50 & $\downarrow$ & $62.2 \pm 6.3$ & $\downarrow$ \\
\hline \multirow{3}{*}{ Fungal } & $3-\tau$ & 3.65 & 0.72 & $\downarrow$ & $\downarrow$ & $80.3 \pm 4.4$ & $\downarrow$ & 56.5 & $\downarrow$ & $44.7 \pm 2.1$ & $\downarrow$ \\
\hline & $4-\tau$ & 3.65 & 1.99 & 1.99 & 0.72 & $45.5 \pm 7.2$ & $68.9 \pm 9.2$ & 56.5 & 51.0 & $58.2 \pm 2.8$ & $46.4 \pm 3.3$ \\
\hline & Av erage & 2.79 & 1.69 & $\downarrow$ & $\downarrow$ & $39.4 \pm 8.0$ & $\downarrow$ & 54 & $\downarrow$ & $58.0 \pm 3.2$ & $\downarrow$ \\
\hline
\end{tabular}




\section{Figure Legends}

Figure $1 \quad$ FLIM maps from TSCSPC. Lifetime maps are shown for fixed mammalian cells expressing CFP (a) or a CFP-YFP fusion protein (b), and for living fungal cells expressing Cerulean (c) or a Cerulean-Venus fusion protein (d). The fungal cells continued to grow throughout the period of data acquisition. The lifetime refers to the CFP emission. The scale bar is $50 \mu \mathrm{m}$ for all images.

Figure 2 TSCSPC images and fluorescence decays from selected regions for fixed mammalian cells expressing CFP (a) or a CFP-YFP fusion protein (b), and for living fungal cells expressing Cerulean (c) or a Cerulean-Venus fusion protein (d). The decays have been normalized to allow direct comparison. The fungal cells continued to grow throughout the period of data acquisition. The scale bar is $50 \mu \mathrm{m}$ for all images.

Figure3 Comparison of the 2- and 3-exponential fits of the CFP-YFP TSCSPC data in fixed mammalian cells. The structure in the residuals of the 2-exponential fit disappears upon adding a third component and the reduced $\chi^{2}$ parameter decreases from 1.472 to 1.034.

Figure 4 Observation of YFP rise time. TSCSPC of a fixed mammalian cell expressing CFP-YFP with detection of either the CFP emission (a) or the YFP emission (b). The YFP fluorescence displays a rise time of ca. 500ps, which is indicative of FRET from the CFP. The region of analysis is the same for both cells and is highlighted in yellow.

Figure 5 Schematic model of photophysical processes involved in FRET for cells expressing a cyan protein or cyan-yellow fusion protein. (a) The cyan donor is in an equilibrium between two conformations ( $\mathrm{D}_{1}$ and $\mathrm{D}_{2}$ ). The cyan donors absorb blue light and has cyan emission, with each conformation having a unique fluorescence lifetime $\left(\tau_{\mathrm{D} 1}\right.$ or $\left.\tau_{\mathrm{D} 2}\right)$. The yellow protein is in an equilibrium between non-protonated $\left(\mathrm{AC}_{1}\right)$ and protonated states $\left(\mathrm{AC}_{2}\right)$. Only $\mathrm{AC}_{1}$ acts as FRET acceptor of cyan light, producing yellow emission with lifetime, $\tau_{\mathrm{Al}}$.(b) In the $3 \tau$ model, FRET only occurs between one of the cyan conformations and the yellow acceptor, giving three unique lifetimes. (c) In the $4 \tau$ model, both cyan conformations participate in FRET, resulting in four unique lifetimes. Since our data is well fitted by three lifetime components, this model assumes that two of the lifetimes $\left(\tau_{\mathrm{D} 2}\right.$ and $\left.\tau_{\mathrm{DAl}}\right)$ are similar and cannot be resolved. 
FIGURE 1

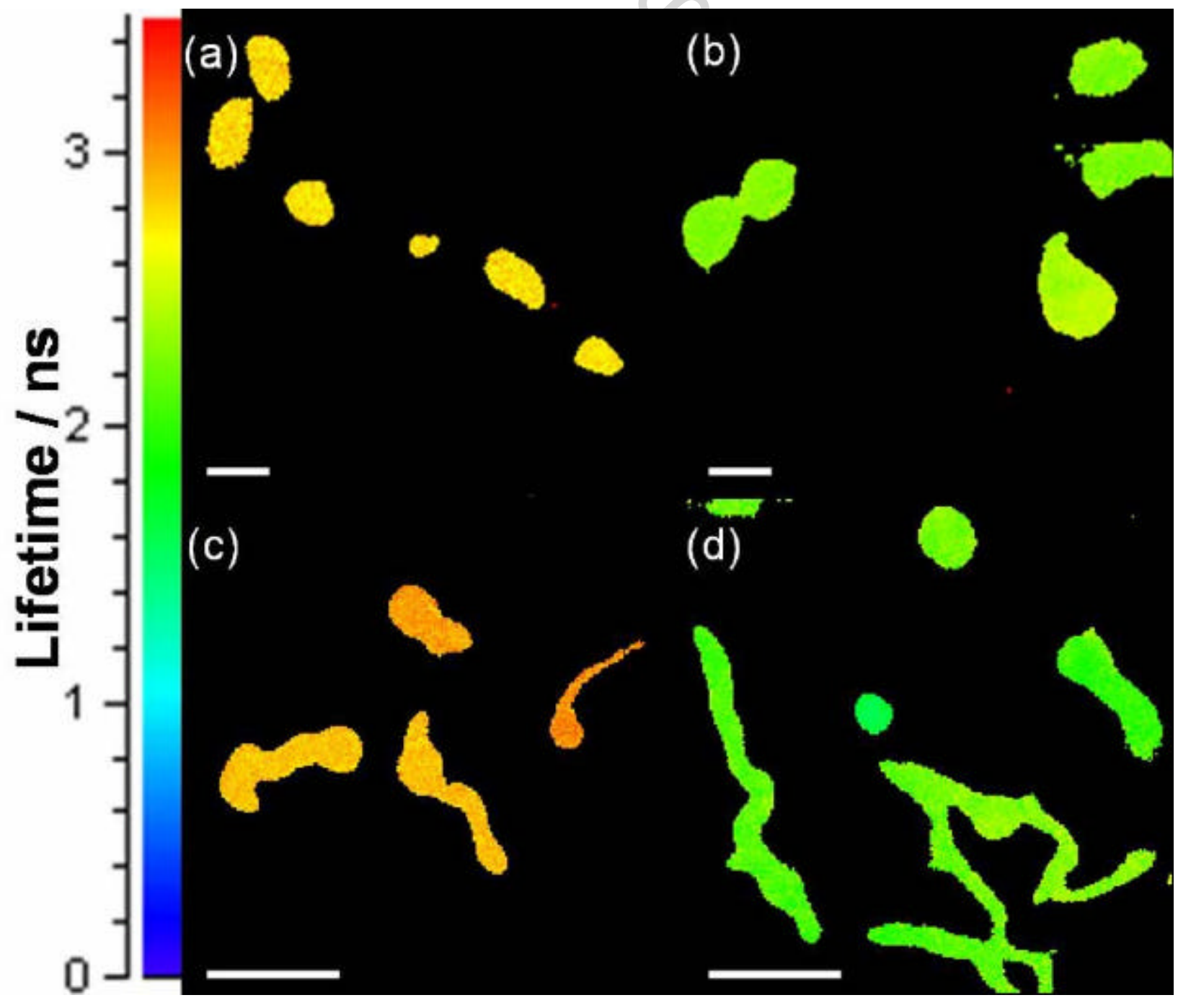


FIGURE 2
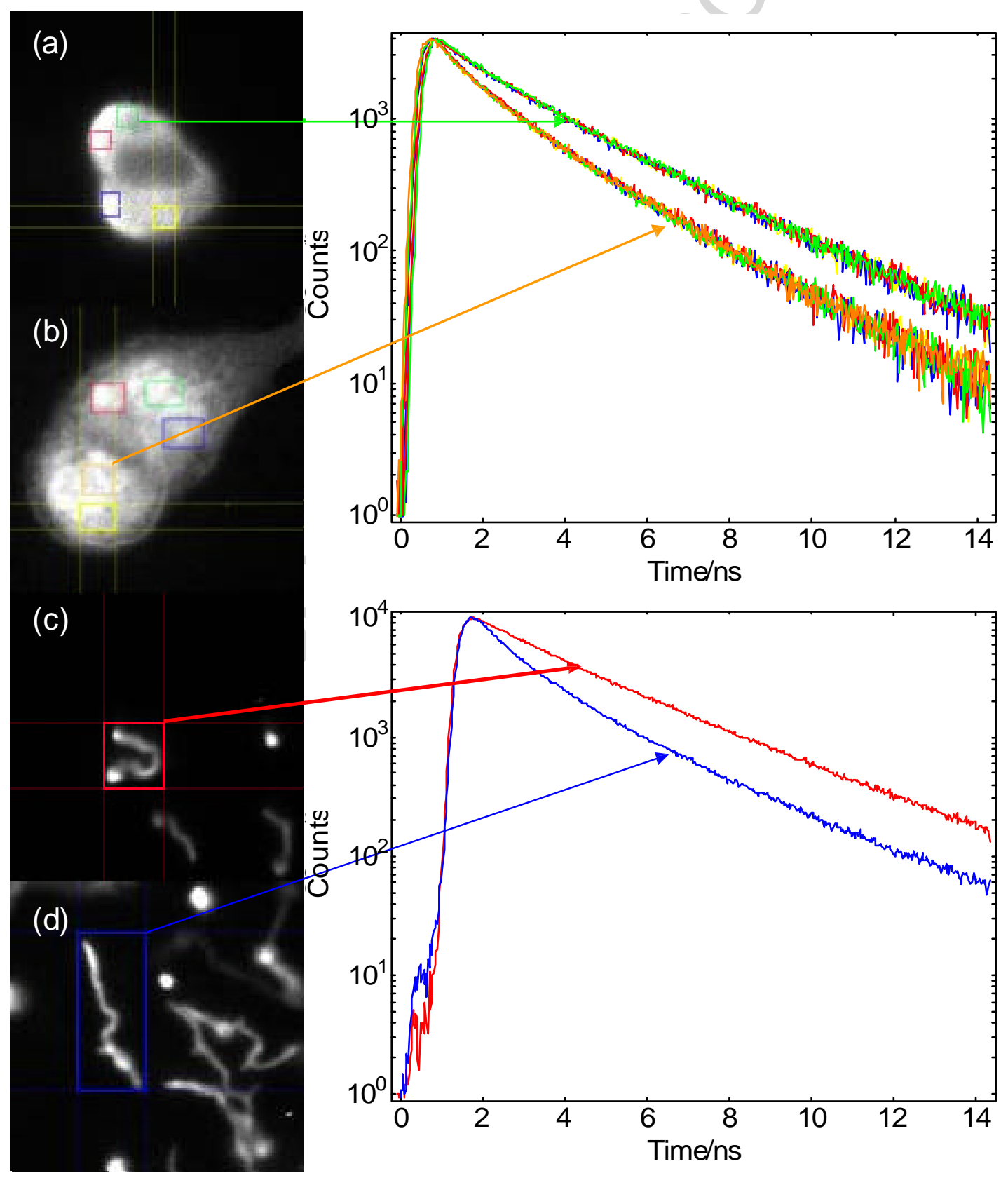


\section{FIGURE 3}
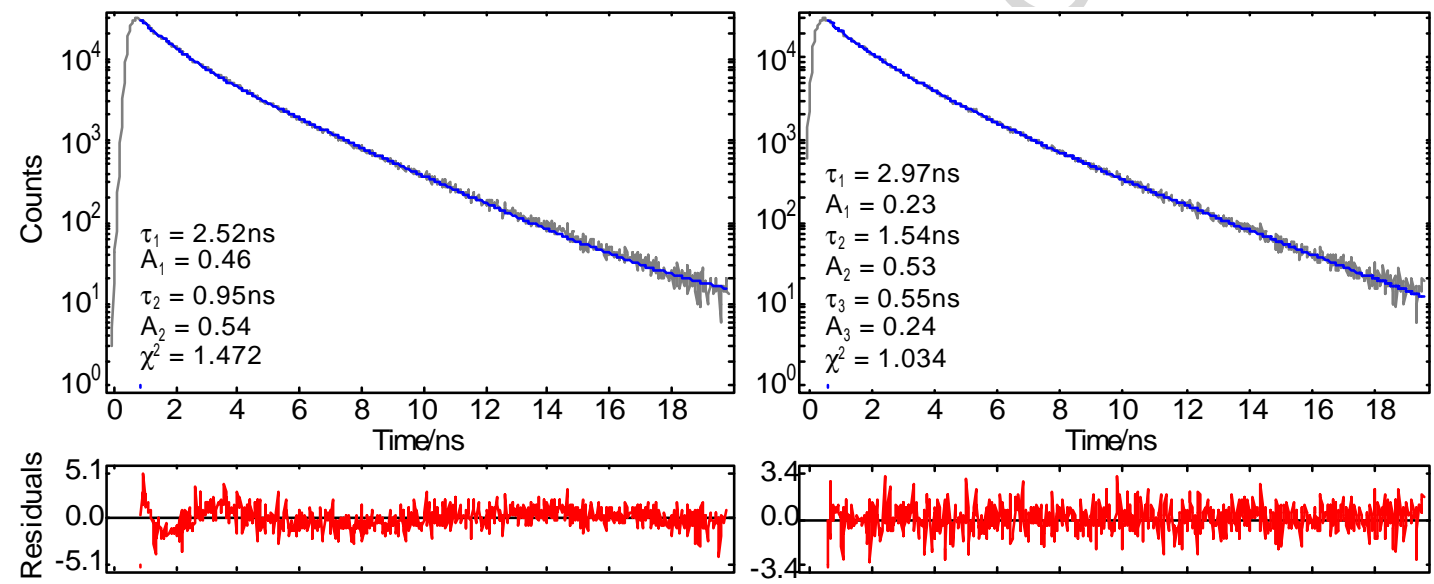
FIGURE 4
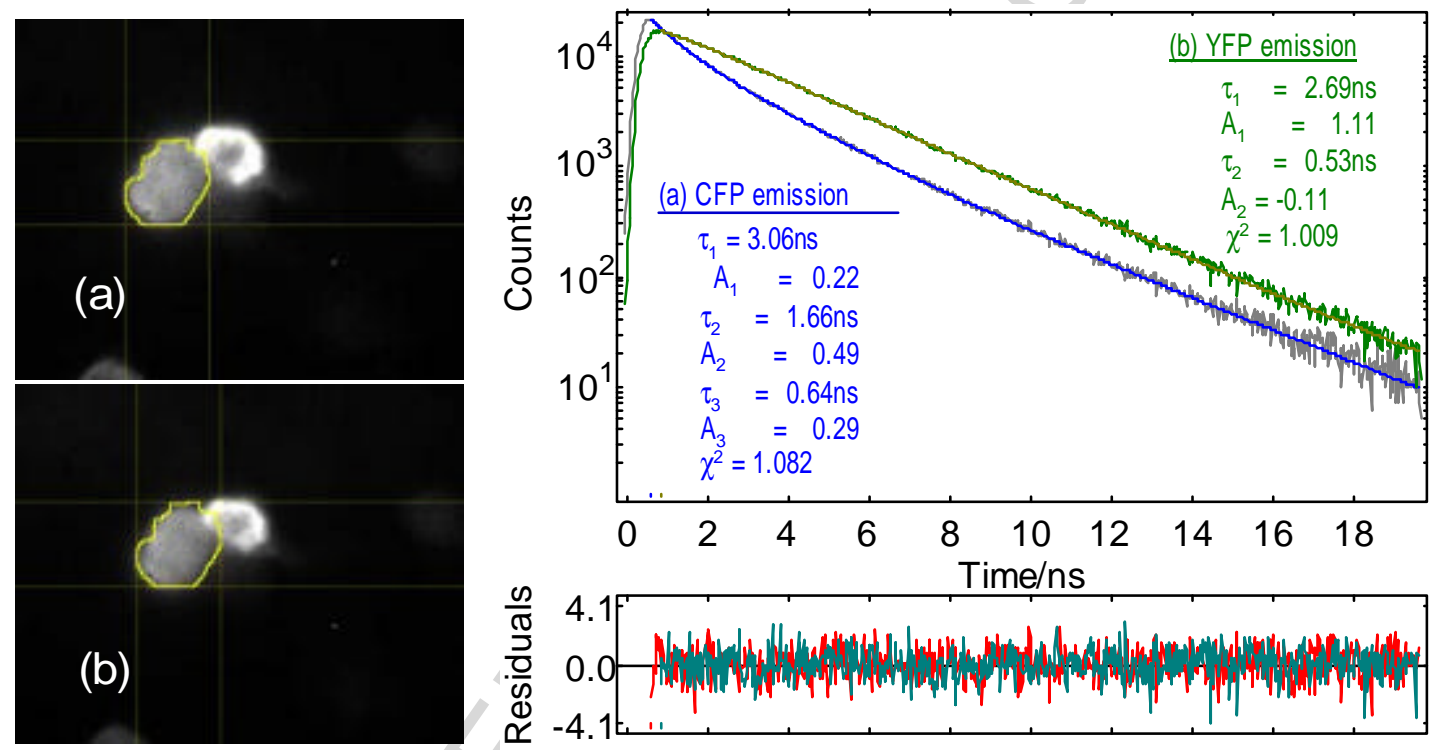

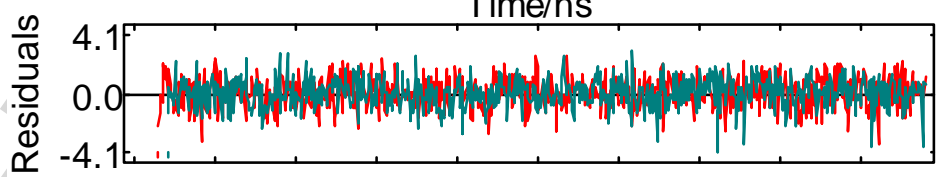




\section{FIGURE 5}

(a)
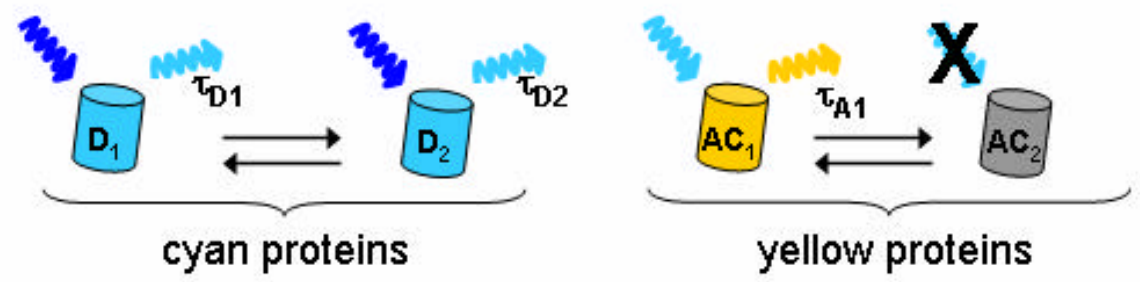

(b)
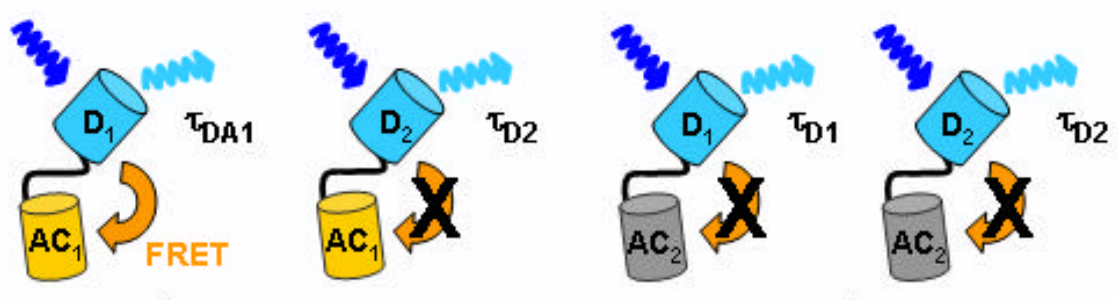

Model $1-3 \tau$

(c)

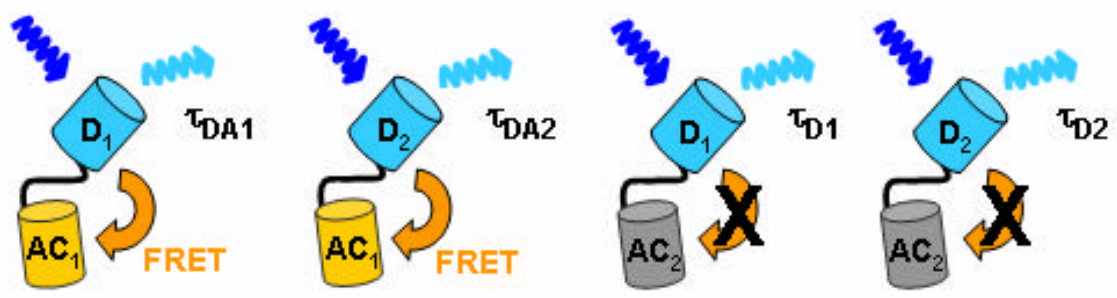

Model $2-4 \tau$ 